\title{
Uniparental Disomy in Somatic Mosaicism 45,X/46,XY/46,XX Associated with Ambiguous Genitalia
}

\author{
Alexandre Serra ${ }^{a}$ Friederike Denzer ${ }^{b}$ Olaf Hiort ${ }^{g}$ Thomas F. Barth ${ }^{c}$ \\ Doris Henne-Bruns $^{d}$ Gotthold Barbi ${ }^{e}$ Günther Rettenberger ${ }^{f}$ \\ Martin Wabitsch ${ }^{\mathrm{b}}$ Walter Just ${ }^{\mathrm{e}}$ Clothilde Leriche $^{\mathrm{a}}$ \\ ${ }^{a}$ Division of Pediatric Surgery, Department of Surgery, and Departments of b Pediatric Endocrinology, ${ }^{c}$ Pathology \\ and ${ }^{d}$ Visceral and General Surgery, and ${ }^{\mathrm{e}}$ Institute of Human Genetics, University of UIm, Ulm, ${ }^{\mathrm{f}}$ Genetikum GmbH, \\ Neu-Ulm, and ${ }^{9}$ Division of Experimental Pediatric Endocrinology and Diabetology, Department of Pediatrics, \\ University of Lübeck, Lübeck, Germany
}

\section{Key Words}

Ambiguous genitalia - Disorders of sex development .

Somatic mosaicism 45,X/46,XY/46,XX - Uniparental disomy

\begin{abstract}
Disorders of sex development (DSD) affect the development of chromosomal, gonadal and/or anatomical sex. We analyzed a patient with ambiguous genitalia aiming to correlate the genetic findings with the phenotype. Blood and tissue samples from a male patient with penoscrotal hypospadias were analyzed by immunohistochemistry, karyotyping and FISH. DNA was sequenced for the $A R, S R Y$ and $D H H$ genes, and further 26 loci in different sex chromosomes were analyzed by MLPA. The gonosomal origin was evaluated by simple tandem repeat (STR) analysis and SNP array. Histopathology revealed a streak gonad, a fallopian tube and a rudimentary uterus, positive for placental alkaline phosphatase, cytokeratin-7 and c-kit, and negative for estrogen, androgen and progesterone receptors, alpha-inhibin, alpha-1-fetoprotein, $\beta$-hCG, and oct-4. Karyotyping showed a 45,X/46,XY mosaicism, yet FISH showed both $46, \mathrm{XX} / 46, \mathrm{XY}$ mosaicism (gonad and urethral plate), 46,XX (uterus and tube) and
\end{abstract}

(c) 2015 S. Karger AG, Base

$1661-5425 / 15 / 0093-0136 \$ 39.50 / 0$
46,XY karyotypes (rudimentary testicular tissue). DNA sequencing revealed intact sequences in SOX9, WNT4, NROB1, NR5A1, CYP21A2, SRY, AR, and DHH. STR analysis showed only one maternal allele for all $X$ chromosome markers (uniparental isodisomy, UPD), with a weaker $S R Y$ signal and a 4:1 ratio in the $X: Y$ signal. Our findings suggest that the observed complex DSD phenotype is the result of somatic gonosomal mosaicism and UPD despite a normal blood karyotype. The presence of UPD warrants adequate genetic counseling for the family and frequent, lifelong, preventive follow-up controls in the patient.

(c) 2015 S. Karger AG, Basel

The definition of 'disorders of sex development' (DSD, MeSH: D012734) includes a broad spectrum of genetic and endocrine abnormalities which profoundly impact the gender differentiation in affected children. Accordingly, the management of children with DSD is a challenge for all professionals involved, most importantly before life-changing decisions regarding gender definition and subsequent operative procedures (as soon as the child has reached an adequate age for informed consent, ac-

\section{KARGER 125}

E-Mail karger@karger.com www.karger.com/sxd
Alexandre Serra, MD, $\mathrm{PhD}$

Division of Pediatric Surgery, Department of Surgery University of Ulm

Eythstrasse 24, DE-89075 Ulm (Germany)

E-Mail Alexandre.serra@uniklinik-ulm.de 
cording to the latest guidelines) are to be considered. More recently, it has been shown that the incidence of DSD is increasing due to more successful in vitro fertilization procedures and multiple pregnancies [Plachot and Crozet, 1992; Simon-Bouy et al., 2003].

Generally speaking, ovotesticular disorders of sex development or gonadal dysgenesis are a defective gonadal development with a wide spectrum of chromosomal mosaic variants, whose phenotype may range from female to male with several types of alterations in the gonads and the internal and external genitalia [Repas-Humpe et al., 1999], also an essential information to be investigated prior to any therapy. However, in many cases, the diagnosis relying purely on blood samples does not reveal the underlying genetic variation responsible for the phenotype, especially when a somatic chromosomal variant is present. Further, cases of somatic mosaicism as a result of acquired postzygotic uniparental disomy (UPD) during mitosis (i.e. the presence of 2 copies of a particular chromosome from the same parent, either maternal or paternal, and no copies of this chromosome from the other parent) may be associated with an increased risk of tumor development [Tuna et al., 2009].

Accordingly, in the present manuscript, we analyze a DSD patient with severe intersex aiming to correlate the genetic findings of blood and tissue samples (uterus, urethra plate) with the phenotype. We emphasize the necessity of the identification of a UPD in DSD patients with mosaics and chimera, thus alerting for the increased tumor development risk due to the presence of a somatic mosaicism with unbalanced gonosome dosage of unknown genealogy.

\section{Clinical Summary}

The phenotypically male patient was referred to the Departments of Pediatric Surgery and Pediatric Endocrinology of the University of Ulm due to a severe penoscrotal hypospadias and undescended testis. The child had a urethral meatus on the proximal penis shaft. The left testicle was descended and well-developed; the right testicle could not be palpated either in the scrotum or in the inguinal area (fig. 1).

Laboratory evaluations revealed a slightly decreased anti-Müllerian hormone $(49.7 \mu \mathrm{g} / \mathrm{l}$, reference range $60.86-78.86)$ and normal inhibin-B (99 ng/l, reference range 35-182). These findings were suggestive of the presence of functional testicular tissue. Testosterone and estradiol were not detectable, consistent with the prepubertal stage. Levels for androstenedione, dehydroepiandrosterone sulfate, FSH, LH, and cortisol and adrenocorticotropic hormone were within the normal range for prepubertal males. The $\beta$-human chorionic gonadotropin ( $\beta$-hCG) stimulation test, performed with $\beta$-hCG $5,000 \mathrm{IE} / \mathrm{m}^{2}$ injected intramuscularly, showed a normal testosterone
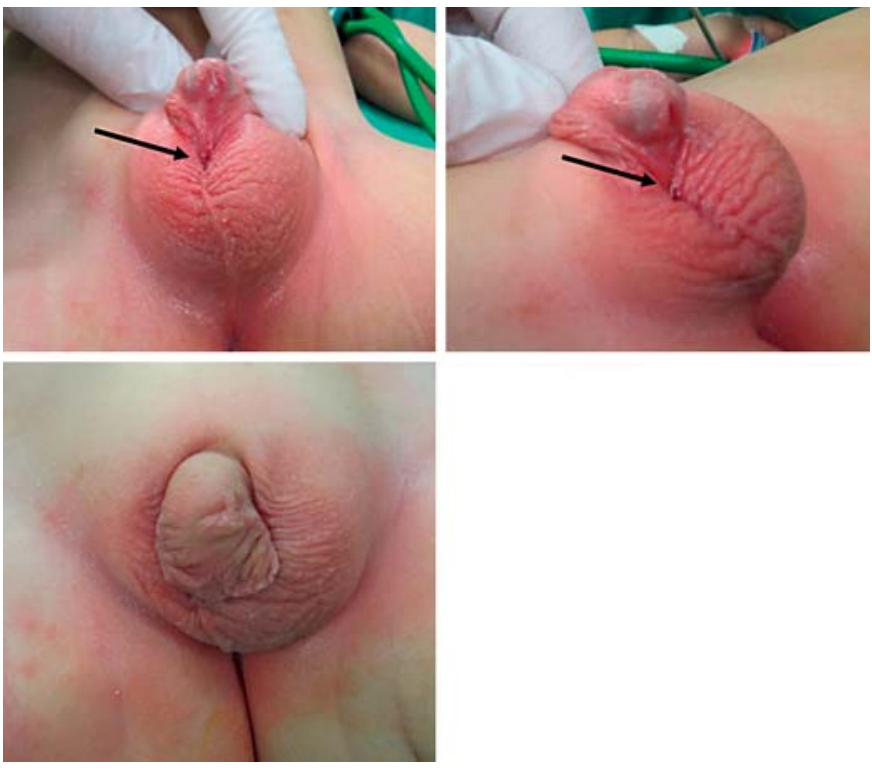

Fig. 1. Preoperative images of the external genitalia showing the severe penoscrotal hypospadia and undescended testis on the right side. The urethral meatus (arrow) is located proximally in the penis shaft; the left testicle was well-developed and located in the scrotum, while the right testicle could not be palpated either in the scrotum or in the inguinal area.
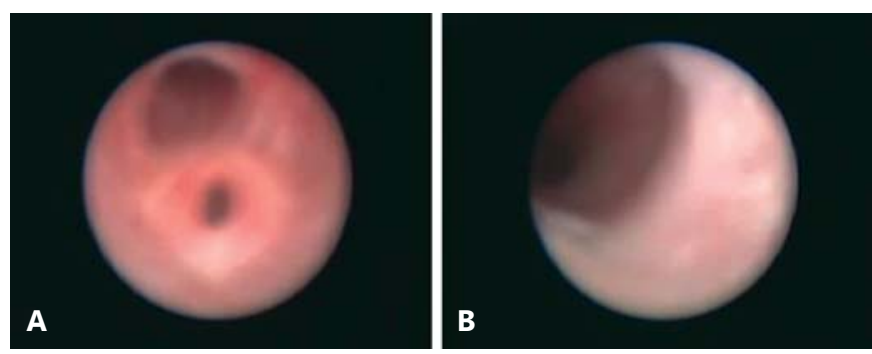

Fig. 2. Urethrocystoscopy revealing a normal bladder with orthotopic ureters, a third ostium in the pars prostatica (A) and a rudimentary vagina linked to a rudimentary uterus and open fallopian tube (B).

level on day 3 (2.07 $\mu \mathrm{g} / \mathrm{l}$, reference range for prepubertal males $<10$ $\mu \mathrm{g} / \mathrm{l})$, along with a normal androstenedione:testosterone ratio and a normal testosterone:dihydrotestosterone ratio. These results were also consistent with the presence of functional testicular tissue and ruled out both $5 \alpha$-reductase deficiency and $17 \beta$-hydroxysteroiddehydrogenase deficiency. Additionally, a human menopausal gonadotropin (HMG) stimulation test was performed with $150 \mathrm{IE}$ HMG, injected subcutaneously, on 3 following days, and since estradiol was not detectable before or after stimulation on the fourth day, the presence of functional ovarian tissue was unlikely.

Further diagnostics included cystoscopy and laparoscopy under general anesthesia at the age of 3 months. The urethrocystoscopy (fig. 2) revealed a normal bladder with orthotopic ureters. In 
the pars prostatica, there was a third ostium, which could be filled with contrast medium and the X-ray revealed a dilatation compatible with a rudimentary vagina linked to a uterus. Additionally, there was a passage of contrast medium in the abdominal cavity, suggesting an open fallopian tube.

The laparoscopy confirmed the findings of a rudimentary uterus, a rudimentary fallopian tube, and a streak gonad on the right side. A structure reminiscent of a round ligament connecting the uterus with the urethra at the level of the prostate was also seen. These structures were removed 'en bloc' and sent to histopathological examination. Additionally, a small ductus deferens was observed on the left side, yet the patient had no prostate.

Before the hypospadias repair, the patient received local applications of dihydrotestosterone gel to enlarge the phallus. The surgical correction of the hypospadias was carried out at the age of 8 months and consisted of the reconstruction of the urethra using a preputial skin flap after correcting the penile deviation (due to a partial atresia of the corpora cavernosa) (fig. 3). Postoperatively, the patient was able to void spontaneously through the distally located urethral meatus. The follow-up visits showed normal growth and development with regular voiding and normal spontaneous erection (as observed by the parents).

Informed written consent of the parents including permission for further genetic studies of blood and tissue samples was obtained in accordance with the guidelines of the Ethics Commission of the University of Ulm, Germany.

\section{Materials and Methods}

\section{Histopathology}

For histopathological examination, formalin-fixed and paraffin-embedded sections of the surgical samples were stained with hematoxylin and eosin according to standard protocols.

\section{Immunohistochemistry}

Paraffin sections of about $2 \mu \mathrm{m}$ were obtained and stained according to standard protocols. Briefly, monoclonal and/or polyclonal antibody-specific serum [placental alkaline phosphatase (PLAP); cytokeratin-7; c-kit; estrogen, androgen, and progesterone receptors; alpha-inhibin; alpha-1-fetoprotein; $\beta$-hCG; oct-4, and Mib-1 (Medac, Wesel, Germany)] was diluted 1:100 in PBS and $100 \mu \mathrm{l}$ were incubated on deparaffined slides. Slides were pretreated for antigen retrieval (microwave oven for $20 \mathrm{~min}$ in citrate buffer $20 \mathrm{~mm}, \mathrm{pH} 6$ ). As detection system, we used the Dako detection kit K5005 (Dako, Glostrup, Denmark).

\section{Karyotyping}

Cytogenetic banding analyses were performed on PHA-stimulated lymphocytes from peripheral blood cultured for $72 \mathrm{~h}$ as well as on cultured skin fibroblasts after GTG-banding according to standard techniques.

\section{Androgen Receptor Sequencing}

The whole coding region of the androgen receptor gene, including intron/exon boundaries, was amplified from blood leukocyte-derived DNA by PCR and subsequently sequenced according to standard procedures using an automated fluorescence-based sequencer (ABI 3100, Perkin-Elmer, Weiterstadt, Germany).

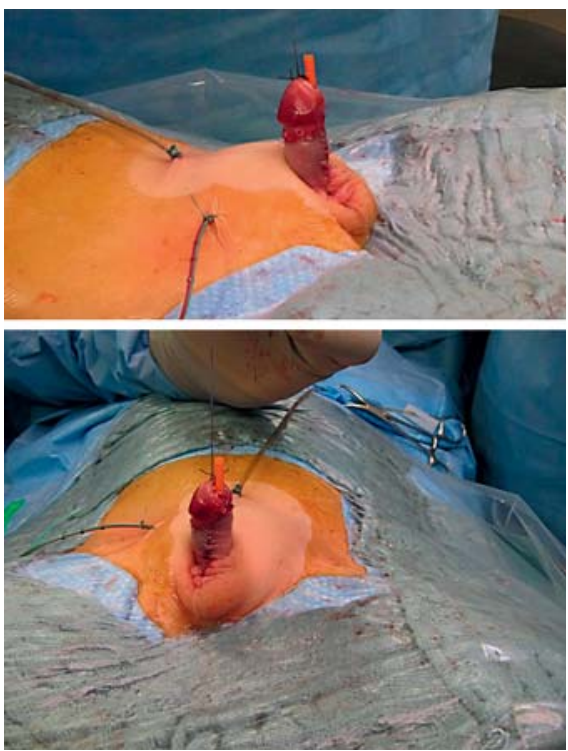

Fig. 3. Postoperative images after hypospadias correction using a modified Dukett technique.

\section{SRY and DHH Sequencing}

DNA from peripheral blood lymphocytes was extracted according to standard techniques. A total of 26 loci in different chromosomes were analyzed by MLPA (Probe set P185-B1, MRC Holland). Additional sequencing of the SRY gene (ENSEMBLENST00000383070) and all exons of the DHH gene (ENSEMBLENST00000266991) was performed.

\section{SNP-CGH Array Analysis}

DNA from the patient and his parents was isolated from EDTA blood as well as DNA from the paraffin-embedded tissue of streak gonads from the patient with the Roche High Pure PCR template prep kit (Roche, Mannheim, Germany) according to the manufacturer's protocol.

Total genomic DNA was directly labeled against $500 \mathrm{ng}$ of $\mathrm{Hu}-$ man male genomic reference DNA (Coriell Institute, Camden, N.J., USA; NA12891 male) and 500 ng DNA of the patient using the Agilent Genomic DNA enzymatic labeling Kit (p/n 5190-0049) according to Agilent standard CGH+SNP protocol (version 4.0). The SurePrint Human Genome CGH+SNP 180K microarrays (Agilent Technologies G4890A, Santa Clara, Calif., USA) were hybridized and washed using the Agilent aCGH Wash Kit (p/n 51885226) according to the manufacturer's protocol and scanned using an Agilent dual laser DNA microarray scanner. Data were extracted using Agilent Feature Extraction (version 9.5) software and analyzed using Agilent DNA Analytics (version 4.0) software.

\section{Short Tandem Repeat Analysis and Amelogenin}

$100 \mathrm{ng}$ DNA from the patient and his parents each were amplified in multiplex PCR reactions, which have been set up in a multiplex PCR with primers for STR loci DXS8378, DXS6809, DXS10160, DXS8377, AMELX/Y, and SRY. Primer sequences for the DXS loci have been taken from the ChrX-STR.org 2.0 database: http://xdb.qualitype.de/xdb/linkageTable.jsf. 

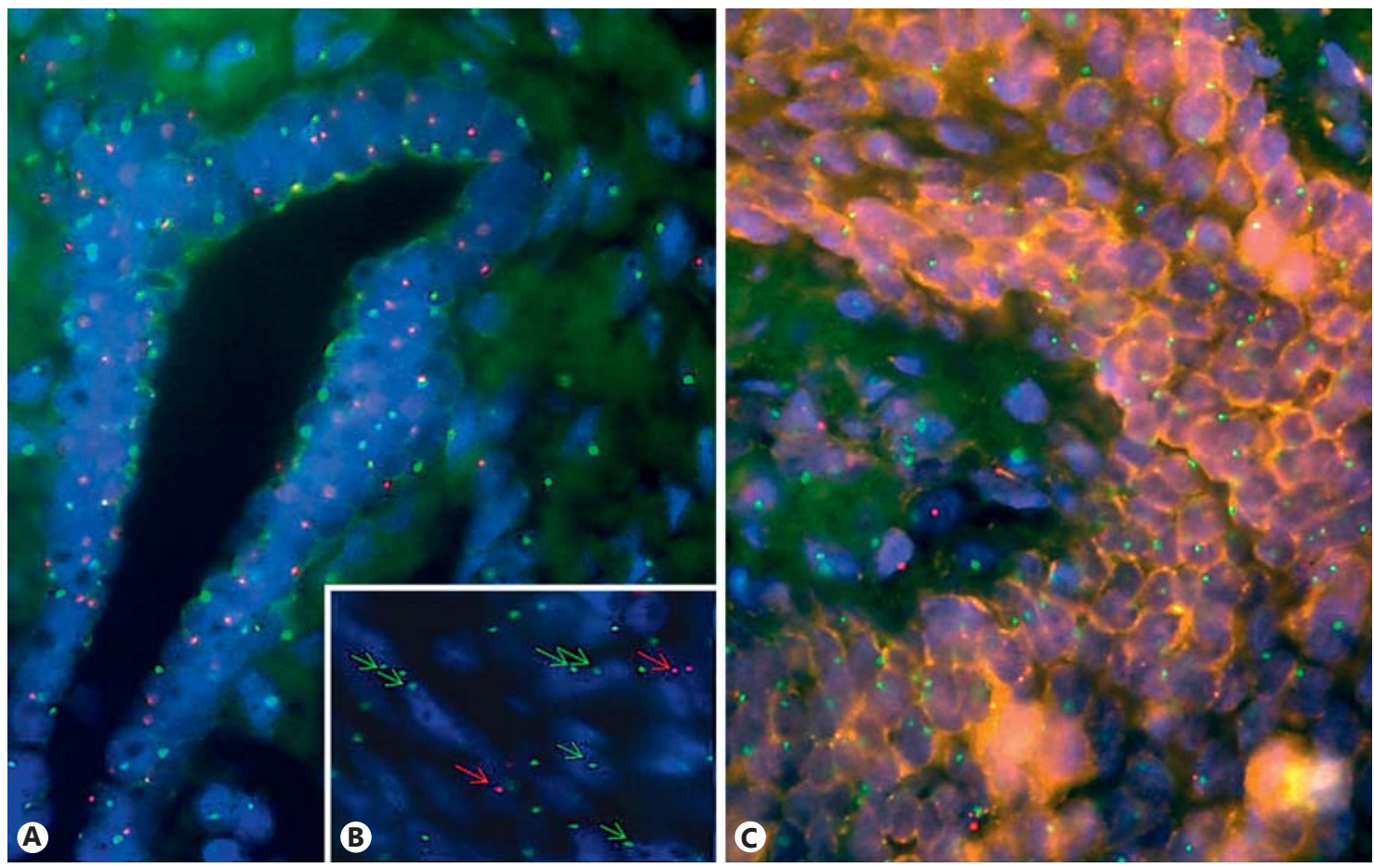

Fig. 4. FISH analysis of the gonadal structure and the resected urethral plate. X chromosomes are stained in green; $\mathrm{Y}$ chromosomes are stained in red (arrows in B). A Uterine tissue with predominantly $(90 \%)$ XX in the spindle cells mixed with some XY cells, probably lymphocytes. B PLAP-positive segments of rudimentary testicular tissue with mostly (80-90\%) XY cells, confirming the gonosome mosaicism in the streak gonad. C Highly atrophied urethral plate with mixed XX and XY cells.
Primer sequences for amelogenin and SRY have been taken from the NCBI database. Amplifications for all primers have been performed with the Universal Multiplex Cycling Protocol for the QIAGEN Multiplex PCR Kit (cat 206143, Qiagen, Hilden, Germany) according to the manufacturer's 'Universal Multiplex Cycling protocol'.

Amplification products were analyzed with a genetic analyzer ABI 310 (Life Technologies, Carlsbad, Calif., USA) with Genscan software v3.7.1.

\section{Results}

Histopathology of the streak gonad revealed a fallopian tube and the stroma of a rudimentary uterus, together with a tubular structure which was positive in immunohistochemistry for PLAP, cytokeratin-7 and c-kit, and negative for estrogen, androgen and progesterone receptors, alpha-inhibin, alpha-1-fetoprotein, $\beta$-hCG, and oct4. The proliferation rate measured by Mib-1 antibodies was slightly increased in the c-kit-positive cells.

Conventional banding analysis of lymphocyte cultures from peripheral blood samples showed a mosaic consist- ing of a cell line with a normal male karyotype (46,XY; observed in 85 metaphases) and of a cell line with monosomy X (45,X; represented by 9 metaphases). In cultured skin fibroblasts, the mosaic was represented by 153 metaphases with monosomy $\mathrm{X}$ and only 23 metaphases with a normal 46,XY karyotype. Similar results were seen by interphase FISH examination of both the gonadal structure and of the resected urethral plate. However, nuclei of spindle cells in the uterine tissue were predominantly (90\%) XX (the same as in the epithelium of the fallopian tube) mixed with some XY cells, possibly lymphocytes (fig. 4A). The nuclei with XXY (2\%) and XYY (2\%) may represent technical artefacts of the FISH method. In contrast, cells from the PLAP-positive segments of rudimentary testicular tissue were mostly (80-90\%) XY, confirming a gonosome mosaicism in the streak gonad (fig. 4B). Other nuclei were polysomic [XYY (10\%) and XXYYY (4\%)]. Interestingly, the highly atrophied urethral plate showed mixed XX and XY cells (fig. 4C). The karyotypes in these areas were:

- Uterus area 1: nuc ish(DXZ1,DYZ3) $\times 1[48] /(\mathrm{DXZ1 \times 2)}$ $[42] /(\mathrm{DXZ} 1 \times 2, \mathrm{DYZ} 3 \times 1)[2] /(\mathrm{DXZ} 1 \times 2, \mathrm{DYZ} 3 \times 2)[2] /$ 


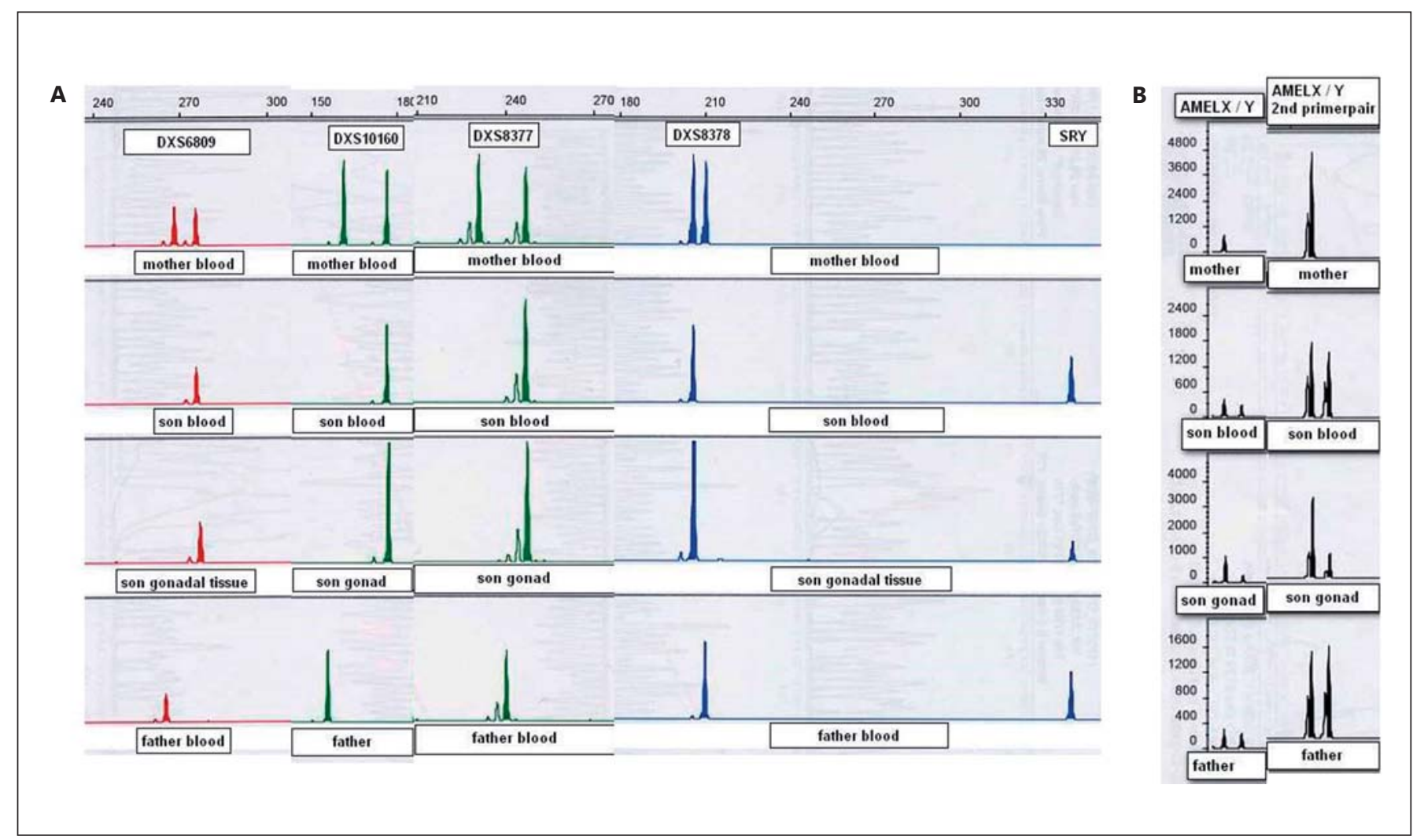

Fig. 5. A STR analyses using probes DXS6809, DXS10160, DXS8377, and SRY showing a marked decrease of the Y signal in the gonads in comparison to blood and to samples from each parent. B Amplification of the X and Y chromosomes from blood and gonad samples of the patient in comparison to his parents using amelogenin primers. A pathological $\mathrm{Y}$ to $\mathrm{X}$ chromosome signal ratio of 1:4 is clearly evident in the probes from the gonads of the patient.
$(\mathrm{DYZ1 \times 2)[6]} \mathrm{(again,} \mathrm{in} \mathrm{this} \mathrm{case} \mathrm{the} \mathrm{YY} \mathrm{might} \mathrm{repre-}$ sent technical artefacts of the FISH method), i.e. 46,XX: 42\%, 46,XY: 48\%, 47,XXY: $2 \%$, and 47,XXYY: $2 \%$.

- Uterus area 2: nucish(DXZ1,DYZ3) $\times 1[56] /(\mathrm{DXZ1 \times 2)}$ [30]/(DXZ1×1,DYZ3×2)[10]/(DXZ1×2,DYZ3×3) [4], i.e. $46, \mathrm{XX}$ : $30 \%, 46, \mathrm{XY}: 56 \%, 47, \mathrm{XYY}: 10 \%$, and 49,XXYYY: $4 \%$.

- Urethra plate area 1: nuc ish(DXZ1,DYZ3) $\times 1[15] /$ (DXZ1×2)[85], i.e. $46, X X: 85 \%$ and $46, X Y: 15 \%$.

- Urethra plate area 2: nuc ish(DXZ1,DYZ3) $\times 1[57] /$ (DXZ1×2)[43], i.e. $46, X X: 43 \%$ and 46,XY: $57 \%$.

- Urethra plate area 3: nuc ish(DXZ1,DYZ3) $\times 1[50] /$ (DXZ1×2)[50], i.e. $46, \mathrm{XX}: 50 \%$ and $46, \mathrm{XY}: 50 \%$.

Sequence analysis of $S R Y$ and $D H H$ revealed no variants or biallelic configuration in loci associated with sex determination, such as SOX9, WNT4, NROB1, NR5A1, CYP21A2, SRY, or DHH.

The analysis of exons 1-8 of the $A R$ gene, including intron flanking sequences, did not show any variants, nei- ther in the normal sequence nor in the glutamine-coding CAG-repeat sequence in exon 1.

The STR analysis showed only one maternal allele for all X chromosome markers, and $S R Y$ testing confirmed a reduction of peak height to $25 \%$ of the Y chromosome signal in the gonadal probe, which further confirmed the mosaicism of the gonosomes. These results are shown in figure 5A. Accordingly, the PCR amplification of the amelogenin loci of the $\mathrm{X}$ and $\mathrm{Y}$ chromosomes revealed a $\mathrm{Y}$ to $\mathrm{X}$ chromosome signal ratio of 1:4 (fig. 5B) in the gonad sample, confirming the STR analysis.

Finally, the SNP array analysis of peripheral blood from the child and both parents showed a total of 1,860 SNPs in the X chromosome, of which 857 could be identified. All of these only had a monoallelic signal without any indication of a second maternal allele, confirming $\mathrm{UPD}$, possibly due to a trisomic rescue (fig. 6). 
Fig. 6. Putative mechanism for UPD formation based on the STR analysis which confirmed the homozygosity of all STR markers for the X chromosome. The most likely mechanism involved was a 'trisomic rescue' of a trisomy of postzygotic origin [based on Grati, 2014].

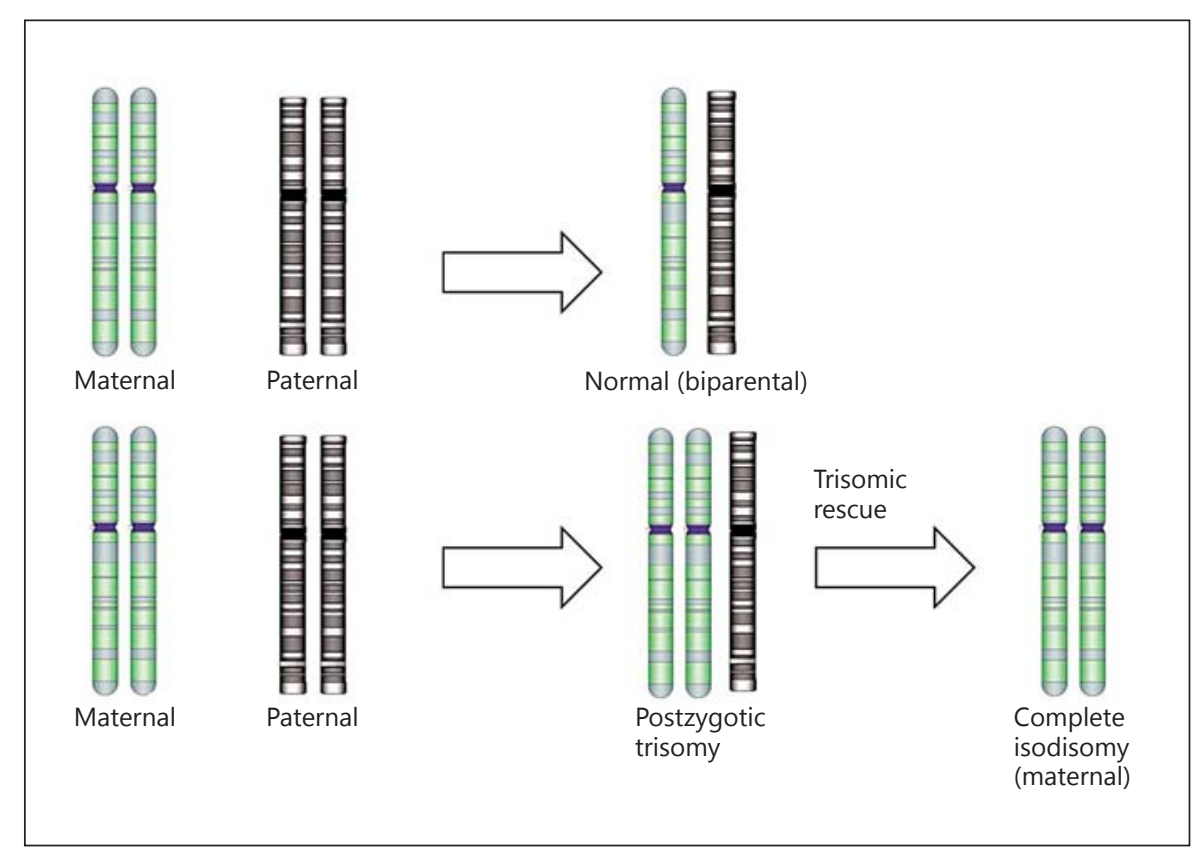

\section{Discussion}

The management of DSD children is challenging for all professionals involved, particularly before definitive decisions regarding gender definition and subsequent operative procedures [Berger-Zaslav et al., 2009]. Accordingly, to assess and define the gender of a child based simply on the phenotype of the internal and external genitalia is insufficient, which warrants a thorough investigation of the genetic background of the affected child and parents in every DSD case [Schoenle et al., 1983].

Mixed cell populations as seen here must be differentiated between a true chimera (defined as the presence of 2 or more cell lines within the same individual but derived from different zygotes) or a mosaic (defined as the presence of more than one cell line within an individual but derived from a single zygote) [Yaron et al., 1999]. Academically, possible mechanisms of chimera formation include: (1) the postzygotic fusion of 2 distinct embryos, yielding a double paternal and a double maternal contribution, also known as tetragametic chimera [Green et al., 1994; Malan et al., 2006, 2007], (2) the fertilization of an ovum and a second polar body by 2 different spermatozoa [Green et al., 1994; Bonthron et al., 1997; Malan et al., 2006], and (3) parthenogenetic division of the oocyte leading to the formation of 2 identical haploid cells and fertilization of each haploid maternal cell by a Y and X sperm (also called parthenogenetic chimera) [Kenner- knecht et al., 1991; Plachot and Crozet, 1992; Malan et al., 2006]. A parthenogenetic chimera was seen in a 5-yearold boy with DSD, in which a $38: 12$ ratio $46, \mathrm{XX} / 46, \mathrm{XY}$ was verified, and microsatellite analysis showed a single haploid ovum dividing parthenogenetically into 2 haploid ova, followed by double fertilization and fusion of the 2 zygotes into a single individual, at the early embryonic stage [Giltay et al., 1998]. More recent reports indicate the same mechanism of chimera formation in a patient with $46, \mathrm{XX} / 47, \mathrm{XY},+14$ chimerism and ambiguous genitalia [Winberg et al., 2010]. In all these cases, SNP array analyses have been recommended to establish the underlying mechanism by identifying the origin of the gonosomes inherited [Shin et al., 2012]. Accordingly, the SNP array analysis ruled out the presence of a chimera in our patient, since only maternally derived X chromosomes were found.

A mosaic on the other hand originates from either: (1) nondisjunction at meiosis 2 (leading to a 24, XX oocyte and then to a 47,XXY zygote after fertilization with a normal 23,Y sperm cell) and the postzygotic loss of one $\mathrm{X}$ chromosome in one cell line and one $\mathrm{Y}$ chromosome in the other cell line during the first mitotic cell division (trisomic rescue) or (2) postzygotic simultaneous mitotic nondisjunction, involving both the $\mathrm{X}$ and $\mathrm{Y}$ chromosomes, in a 46,XY zygote, with death of the nullisomic X cell line [Yaron et al., 1999]. In some cases, mosaicism can result in ambiguous genitalia and DSD with both an ova- 
ry and a testicle, particularly in cases where the $S R Y$ gene is translocated in the short arm of the $\mathrm{X}$ chromosome during the first male meiotic division [Modan-Moses et al., 2003; Grigorescu-Sido et al., 2005; Malan et al., 2007], as observed in a fetus with $46, \mathrm{XY} / 46, \mathrm{XX}$ mosaicism associated with a normal male phenotype [Yaron et al., 1999; Malan et al., 2007]. The distinguishing factor is an intact $S R Y$ gene, which was present in our patient, without mutations or deletions in the sequencing analysis, thus, excluding a 46,XX/45,X mosaic. In some cases, even small cell lines containing $S R Y$ in an ovotestis or rudimentary testicle are responsible for DSD in previously diagnosed $46, \mathrm{XX}$ patients [Nieto et al., 2004], in which SRY fragments may derive from a translocation onto the X chromosome in males with a 46,XX karyotype [GrigorescuSido et al., 2005]. However, the loss of the $S R Y$ gene (such as in a $\mathrm{Y}$ isochromosome) results in a female phenotype despite FISH analysis showing a 46,XY karyotype [Pereira et al., 2008] or a prenatally diagnosed $46, \mathrm{XX} / 46, \mathrm{XY}$ chimera [Binkhorst et al., 2009].

$46, \mathrm{XY} / 46, \mathrm{XX}$ mosaics are more common in Caucasians and present in $\sim 10 \%$ of all DSD patients considered 'true hermaphrodites' [Niu et al., 2002; Alonso et al., 2007]. These DSD patients have common morphological traits, such as a prostatic utricle (pseudovagina) and/or a vagina, cryptorchidism (mostly unilateral) and, generally, a testis on one side and an ovary on the other [Giltay et al., 1998; Repas-Humpe et al., 1999; Yaron et al., 1999] as well as early or late (puberty-onset) defects of the central nervous system [Schoenle et al., 1983; Lieber et al., 1986; Alonso et al., 2007; Souter et al., 2007].

Not only chromosomal aberrations, but also singleand multi-gene variants have been implicated in DSD, such as a homozygous splice-donor-site mutation in the RSPO1 gene (c. $286+1 \mathrm{G}>\mathrm{A})$ leading to a partially functional protein (p.Ile32_Ile95del) in a 46,XX patient [Tomaselli et al., 2008]. In our study, MLPA has ruled out amplifications and/or deletions in the sex-determining genes SOX9, WNT4, NROB1, NR5A1, CYP21A2, SRY, and $D H H$, which eliminated the need for a complete sequencing.

The STR analysis confirmed the homozygosity of all STR markers for the X chromosome, as a consequence of a postzygotic chromosome duplication. Most likely, the mechanism involved was the 'trisomic rescue' of a trisomy of postzygotic origin, constituting a UPD [Souter et al., 2007; Grati, 2014]. The importance of UPD is the increased risk for tumor development due to homozygosity, which is more frequent in cases of acquired and not constitutional UPD, and mostly in autosomes [Tuna et al., 2009]. Specifically, in regards to maternal UPD with $\mathrm{X}$ chromosome isodisomy, as observed in our patient, Tuna et al. [2009] cite an increased risk of sporadic basallike breast cancer, and this was observed in $100 \%$ of $B R C A 1$ (encoding breast cancer 1, early onset)-deficient tumors in one series [Tuna et al., 2009], which definitely warrants an adequate genetic counseling for the patient and the family together with frequent, lifelong, preventive follow-up controls.

In conclusion, the purpose of our descriptive study is to alert the community to complex genetical somatic variants in patients with DSD and ambiguous genitalia which require functional approaches with selected genes and chromosomes to better understand the biology of DSD in children. Further, we could demonstrate that chromosome analyses in peripheral blood lymphocytes alone may not be sufficient to explain the DSD, in which gonosome mosaicism may be seen only in the gonads or other somatic tissues. Most importantly, our study shows that many DSD patients may have a UPD which significantly increases the risk of tumor development. Such patients must undergo lifelong, regular follow-ups and preventive cancer diagnostics in order to minimize the potentially lethal effects of the UPD.
References

\footnotetext{
-Alonso G, Pasqualini T, Busaniche J, Ruiz E, Chemes H: True hermaphroditism in a phenotypic male without ambiguous genitalia: an unusual presentation at puberty. Horm Res 68:261-264 (2007).

- Berger-Zaslav AL, Mehta L, Jacob J, Mercado T, Gadi I, et al: Ovotesticular disorder of sex development (true hermaphroditism). Urology 73:293-296 (2009).

Binkhorst M, de Leeuw N, Otten BJ: A healthy, female chimera with 46,XX/46,XY karyotype. J Pediatr Endocrinol Metab 22:97-102 (2009).
}

Bonthron DT, Strain L, Dean JCS: Amalgamation of in vitro fertilized embryos, resulting in birth of a true hermaphrodite chimera. Am J Hum Genet Suppl 61:A147 (1997).

Giltay JC, Brunt T, Beemer FA, Wit JM, van Amstel HK, et al: Polymorphic detection of a parthenogenetic maternal and double paternal contribution to a $46, \mathrm{XX} / 46, \mathrm{XY}$ hermaphrodite. Am J Hum Genet 62:937-940 (1998).

Grati F: Chromosomal mosaicism in human fetoplacental development: implications for prenatal diagnosis. J Clin Med 3:809-837 (2014). 
Green AJ, Barton DE, Jenks P, Pearson J, Yates JRW: Chimaerism shown by cytogenetics and DNA polymorphism analysis. J Med Genet 31:816-817 (1994)

-Grigorescu-Sido A, Heinrich U, Grigorescu-Sido P, Jauch A, Hager HD, et al: Three new 46,XX male patients: a clinical, cytogenetic and molecular analysis. J Pediatr Endocrinol Metab 18:197-203 (2005).

-Kennerknecht I, Barbi G, Djalali M, Just W, Vogel W, Terinde R: Uncommon chromosomal mosaicism in chorionic villi. Prenat Diagn 11: 569-575 (1991).

Lieber C, Bordiuk J, Desposito F: 46,XY/46,XX blood chimerism with severe central nervous system defect and multiple congenital malformations. Am J Med Genet 23:833-836 (1986).

-Malan V, Vekemans M, Turleau C: Chimera and other fertilization errors. Clin Genet 70:363373 (2006).

-Malan V, Gesny R, Morichon-Delvallez N, Aubry $\mathrm{MC}$, Benachi A, et al: Prenatal diagnosis and normal outcome of a $46, \mathrm{XX} / 46, \mathrm{XY}$ chimera: a case report. Hum Reprod 22:1037-1041 (2007).

-Modan-Moses D, Litmanovitch T, Rienstein S, Meyerovitch J, Goldman B, Aviram-Goldring A: True hermaphroditism with ambiguous genitalia due to a complicated mosaic karyo- type: clinical features, cytogenetic findings, and literature review. Am J Med Genet A 116A:300-303 (2003).

Nieto K, Peña R, Palma I, Dorantes LM, Eraña L, et al: $45, \mathrm{X} / 47, \mathrm{XXX} / 47, \mathrm{XX}, \operatorname{del}(\mathrm{Y})(\mathrm{p}$ ?)/46,XX mosaicism causing true hermaphroditism. Am J Med Genet A 130A:311-314 (2004).

Niu DM, Pan CC, Lin CY, Hwang BT, Chung MY: Mosaic or chimera? Revisiting an old hypothesis about the cause of the 46,XX/ 46,XY hermaphrodite. J Pediatr 140:732-735 (2002).

Pereira SRF, Pereira ACN, Souza MTV, Ramos MRB: FISH, PCR and cytogenetic characterization in a girl with ambiguous genitalia and karyotype mos46,X,iso(Y)(qter $\rightarrow$ p11.3::p11.3 $\rightarrow$ qter $)[80] / 45, \mathrm{X}[17] / 46, \mathrm{X},+\operatorname{mar}[3]$. Genet Mol Res 7:1089-1096 (2008).

Plachot M, Crozet N: Fertilization abnormalities in human in-vitro fertilization. Hum Reprod 7 Suppl 1:89-94 (1992).

Repas-Humpe LM, Humpe A, Lynen R, Glock B, Dauber EM, et al: A dispermic chimerism in a 2-year-old Caucasian boy. Ann Hematol 78: 431-434 (1999).

Schoenle E, Schmid W, Schinzel A, Mahler M, Ritter M, et al: $46, \mathrm{XX} / 46, \mathrm{XY}$ chimerism in a phenotypically normal man. Hum Genet 64: 86-89 (1983).
Shin SY, Yoo HW, Lee BH, Kim KS, Seo EJ: Identification of the mechanism underlying a human chimera by SNP array analysis. Am J Med Genet Part A 158A:2119-2123 (2012).

Simon-Bouy B, Plachot M, Mokdad A, Lavaud N, Muti C, et al: Possible human chimera detected prenatally after in vitro fertilization: a case report. Prenat Diagn 23:935-937 (2003).

Souter VL, Parisi MA, Nyholt DR, Kapur RP, Henders AK, et al: A case of true hermaphroditism reveals an unusual mechanism of twinning. Hum Genet 121:179-185 (2007).

Tomaselli S, Megiorni F, De Bernardo C, Felici A, Marrocco G, et al: Syndromic true hermaphroditism due to an R-spondin1 (RSPO1) homozygous mutation. Hum Mutat 29:220-226 (2008).

Tuna M, Knuutila S, Mills GB: Uniparental disomy in cancer. Trends Mol Med 15:120-128 (2009).

-Winberg J, Gustavsson P, Lagerstedt-Robinson K, Blennow E, Lundin J, et al: Chimerism resulting from parthenogenetic activation and dispermic fertilization. Am J Med Genet Part A 152A:2277-2286 (2010).

Yaron Y, Feldman B, Kramer RL, Kasperski SB, Vo T, et al: Prenatal diagnosis of 46,XY/46,XX mosaicism: a case report. Am J Med Genet 84: 12-14 (1999). 